\title{
Mer Tyrosine Kinase Regulates Disseminated Prostate Cancer Cellular Dormancy ${ }^{\dagger}$
}

Frank Cackowski ${ }^{1,2}$, Matthew R. Eber ${ }^{1,3}$, James Rhee ${ }^{1}$, Ann Decker ${ }^{1}$, Kenji Yumoto $^{1}$, Janice E. Berry ${ }^{\S, 1}$, Eunsohl Lee ${ }^{1}$, Yusuke Shiozawa ${ }^{3}$, Younghun Jung ${ }^{1}$, Julio A. AguirreGhiso $^{4}$, and Russell S. Taichman*,1

${ }^{1}$ Department of Periodontics and Oral Medicine, University of Michigan School of Dentistry, Ann Arbor, MI, USA

${ }^{2}$ Department of Medicine; Division of Hematology \& Oncology, University of Michigan School of Medicine, Ann Arbor, MI, USA

${ }^{3}$ Department of Cancer Biology and Comprehensive Cancer, Wake Forest University

School of Medicine, Winston-Salem, NC, USA

${ }^{4}$ Department of Medicine; Division of Hematology \& Oncology, Tisch Cancer Institute, Department of Otolaryngology, and Black Family Stem Cell Institute, Mt. Sinai School of Medicine, New York, NY, USA

${ }^{\S}$ Deceased on February 4, 2016

*Corresponding author

Russell S. Taichman, D.M.D., D.M.Sc.

University of Michigan School of Dentistry

1011 North University Avenue, Ann Arbor, MI 48109, USA

Phone: 1-734-764-9952

Fax: 1-734-763-5503

email: rtaich@umich.edu

Running Title: MERTK and Prostate Cancer Dormancy

\section{Keywords:}

- MERTK

- AXL

- TYRO3

- prostate cancer

- dormancy

- disseminated tumor cell

Total number of figures and tables: 8

\footnotetext{
$\dagger$ This article has been accepted for publication and undergone full peer review but has not been through the copyediting, typesetting, pagination and proofreading process, which may lead to differences between this version and the Version of Record. Please cite this article as doi: [10.1002/jcb.25768]

Additional Supporting Information may be found in the online version of this article.
}

Received 17 October 2016; Revised Accepted 17 October 2016 Journal of Cellular Biochemistry (C) 2014 Wiley Periodicals, Inc. DOI: $10.1002 / j c b .25768$ 
Financial Support: Direct funding was provided by the NIH/NCI PO1-CA093900, the NIH/NCI Tumor Microenvironment Network U54-CA163124 and supplement, and Department of Defense W81XWH-14-1-0403, W81XWH-15-1-0637, W81XWH-15-10413. R.T. receives support as the Major McKinley Ash Colligate Professor. F.C. received support from the NIH/NCI T32 training grant 5T32CA009357-32 to The University of Michigan Division of Hematology and Oncology. F.C., R.T and Y.J. receive support from The Prostate Cancer Foundation 


\begin{abstract}
Many prostate cancer $(\mathrm{PCa})$ recurrences are thought to be due to reactivation of disseminated tumor cells (DTCs). We previously found a role of the TAM family of receptor tyrosine kinases TYRO3, AXL and MERTK in PCa dormancy regulation.
\end{abstract} However, the mechanism and contributions of the individual TAM receptors is largely unknown. Knockdown of MERTK, but not AXL or TYRO3 by shRNA in PCa cells induced a decreased ratio of P-Erk1/2 to P-p38, increased expression of p27, NR2F1, SOX2, and NANOG, induced higher levels of histone H3K9me3 and H3K27me3, and induced a G1/G0 arrest, all of which are associated with dormancy. Similar effects were also observed with siRNA. Most importantly, knock down of MERTK in PCa cells increased metastasis free survival in an intra-cardiac injection mouse xenograft model. MERTK knockdown also failed to inhibit PCa growth in vitro and subcutaneous growth in vivo, which suggests that MERTK has specificity for dormancy regulation or requires a signal from the PCa microenvironment. The effects of MERTK on the cell cycle and histone methylation were reversed by p38 inhibitor SB203580, which indicates the importance of MAP kinases for MERTK dormancy regulation. Overall, this study shows that MERTK stimulates prostate cancer dormancy escape through a MAP kinase dependent mechanism, also involving p27, pluripotency transcription factors, and histone methylation.

Prostate cancer patients often have long time periods between curative intent surgery or radiation therapy until the time of biochemical recurrence or metastatic disease visible with current imaging, which marks incurable disease with current treatment options. For example, in a large series of patients treated with radical prostatectomy, nearly $20 \%$ 
recurrences occurred at least 5 years after surgery(Amling, Blute et al. 2000). Greater than half of prostate cancer patients with no evidence of disease soon after radical prostatectomy were found to have disseminated prostate tumor cells (DTCs) in their bone marrow, which are thought to be a major source of distant recurrences(Morgan, Lange et al. 2009). This finding implies that many of these tumor cells die, never grow, or grow very slowly. Many investigators refer to this ability of cancer cells to remain viable but not have detectable growth as "cellular dormancy."

There is significant interest in regulators of cancer cellular dormancy. Several studies have identified a low ratio of phosphorylated MAPK3/MAPK1 (Erk 1/2) to phosphorylated MAPK14 (p38) as marking dormant tumor cells. TGFB2 (TGF- $\beta 2$ ) was proposed to be the major ligand responsible for dormant behavior of head and neck squamous cell carcinoma cells. The cell cycle inhibitor CDKN1B (p27) and transcription factor BHLHE41 (DEC2) were implicated as nuclear signals(Bragado, Estrada et al. 2013). More recently, pluripotency associated transcription factors NR2F1, SOX2, SOX9, NANOG, and RARB were identified as transcriptional regulators of dormancy in head and neck, prostate and breast cancers(Sosa, Parikh et al. 2015). Similarly, others have shown that TGF- $\beta$ family member BMP7 maintains prostate cancer dormancy through autocrine SPARC(Sharma, Xing et al. 2016) (Kobayashi, Okuda et al. 2011). A role of the epigenome in regulating cellular dormancy is also becoming apparent. Histone H3 tri-methylated lysine 9 and trimethylated lysine 27 were shown to identify and to be important for dormant cells, primarily in head and neck cancer(Sosa, Parikh et al. 2015). 
Our group has established a role of the TYRO3, AXL and MERTK (TAM) family of receptors and one of their ligands, growth arrest-specific 6 (GAS6), in regulation of prostate cancer cell dormancy in the bone marrow(Shiozawa, Pedersen et al. 2010, Jung, Shiozawa et al. 2012, Taichman, Patel et al. 2013). We also found that GAS6 and MERTK are important for cancer stem like cell formation (Jung, Decker et al. 2016, Shiozawa, Berry et al. 2016). This receptor family has an established role in the regulation of the innate immune system, but more recently has been shown to be important for cancer growth and metastasis as well. For example, MERTK was recently identified in a screen of wild type kinases as a mediator of prostate cancer metastasis(Faltermeier, Drake et al. 2015). The TAM family of receptors have a high degree of homology, but have been shown to have different functions, which might relate to differences in ligand binding affinities and downstream pathways(Graham, DeRyckere et al. 2014). There are at least four vitamin K dependent $\gamma$-carboxyalated protein ligands that bind at least one of the TAM receptors including GAS6, PROS1 (Protein S), TUB (tubby), and TULP1 (tubby like protein 1)(Caberoy, Zhou et al. 2010). We found that GAS6 decreased prostate cancer proliferation and protected the cells from chemotherapy induced apoptosis(Shiozawa, Pedersen et al. 2010, Lee, Decker et al. 2016). We also found that prostate cancer bone metastases grew larger in the absence of GAS6 in a bone(Jung, Shiozawa et al. 2012).

However, these studies did not identify which of the TAM receptors are responsible for the ability of GAS6 to slow prostate cancer growth, while also preventing apoptosis findings which are consistent with cellular dormancy. However, the role of TYRO3 
and MERTK remained unclear. To begin to answer this question, we previously studied the relative expression level TYRO3 and AXL in prostate cancer primary tumors, DTCs and in gross metastases. However, MERTK was not included in these studies. We found that TYRO3 was expressed highly in the primary tumors but that AXL was expressed highly in disseminated but dormant disease(Taichman, Patel et al. 2013). Based on these results, we hypothesized that TYRO3 might play a role when prostate cancer was actively growing but that AXL might play a role when it is dormant. However, these studies did not include experiments to test this hypothesis further than gene expression. Most recently, in work that is currently in press, we reported that $\mathrm{AXL}$ is required for TGF2 to induce prostate cancer dormancy(Yumoto 2016). However, the contributions of MERTK and TYRO3 remain unclear.

In the current study we took an unbiased approach to discern which of the TAM receptors, including MERTK, are required for prostate cancer dormancy escape. With shRNA and siRNA technology, we knocked down the expression of each of the three receptors in three different prostate cancer cell lines. We found that loss of MERTK, but not the other receptors decreased the ratio of P-Erk to P-p38, increased the expression of p27 and pluripotency associated transcription factors, increased the levels of dormancy associated histone H3 marks and caused accumulation of cells in the G1 and G0 phases of the cell cycle, and decreased apoptosis, all of which characterize dormant cells. Importantly the effect of MERTK on the cell cycle and histone $\mathrm{H} 3$ post-translational modifications was reversed by altering MAP kinase signaling with a p38 inhibitor. We also found that MERTK knockdown increased 
metastasis free survival in an intra-cardiac injection mouse prostate cancer xenograft model, but did not inhibit in vitro cell growth or subcutaneous tumor growth showing that it did not compromise global growth characteristics. Thus, our studies implicate MERTK in stimulation of prostate cancer dormancy escape by a mechanism particular to the metastatic microenvironment and involving MAP kinases.

\section{MATERIALS AND METHODS}

\section{Cell culture}

Human PCa cell lines, PC3, Du145, and LNCaP C4-2B (C4-2B) were obtained from American Type Culture Collection (Rockville, MD (PC3 and Du145)) and UroCor (Oklahoma City, OK (C4-2B)). PCa cells were maintained in RPMI 1640 with 10\% fetal bovine serum (FBS) and 1\% penicillin/streptomycin (P/S) in a humidified incubator with $5 \% \mathrm{CO}_{2}$. For in vitro assays, unless indicated otherwise, cells were seeded at a density 1 $\mathrm{x} 10^{5} / \mathrm{ml}$, allowed to rest for one day in $10 \%$ serum and then changed to reduced serum concentrations as indicated. For the p38 inhibitor experiments, cells were first cultured for 14 days under routine conditions with $10 \%$ serum and $5 \mu$ M SB203580 (EMD Millipore \#A8254) dissolved in DMSO or $0.05 \%$ DMSO control.

\section{TAM receptor stable shRNA knockdowns}

GFP and luciferase expressing PCa cell lines (PC3 ${ }^{G F P}$, Du145 ${ }^{G F P}$, and C4-2B ${ }^{G F P}$ cells) were first established by lentiviral transduction. Stable knockdowns of the TAM receptors (TYRO3, AXL and MERTK) were then generated by lentiviral infection. 
Lentiviruses were constructed by the University of Michigan Vector Core using pGIPZ lentiviral vectors containing either a shRNA targeting one of the TAM receptors or a nonsilencing (shControl) shRNA (Open Biosystems). Stable lines were selected with puromycin. Knockdown of greater than $80 \%$ was verified by Western blotting and qRTPCR. qRT-PCR gene expression data is presented as mean \pm SEM of independent cultures.

\section{MerTK transient siRNA knockdowns}

siRNAs targeting MerTK (\# s20474, s20473 and s20472) and control siRNA (siControl) (\# 4390843) were purchased from Thermo-Fisher Scientific. Transient transfection in C42B and PC3 cells was performed using $10 \mathrm{mM}$ of each siRNA with Lipofectamine RNAiMAX reagent (Thermo-Fisher) using the reverse transfection protocol, followed by three days incubation. Knockdown was verified by real time qRT-PCR. Data is presented as mean \pm SEM of triplicate PCR reactions.

\section{Western blotting}

Cells were serum starved overnight unless indicated otherwise. Lysates were prepared in cOmplete lysis M (Roche \#04 719956001$)$ supplemented with proteinase inhibitor Mini cOmplete Tablets (Roche \#04705378) and phosphatase inhibitor PhosSTOP EASYpack Tablets (Roche \#04 906837 001). Protein concentration was determined by the BCA method. $20 \mu \mathrm{g}$ of total protein was added per lane of 4-20\% reducing SDS polyacrylamide Tris-Glycine gels after sample preparation in Laemmli sample buffer. The samples were transferred to PVDF membranes and blocked for 1 hour in 5\% dry milk in TBS with $0.1 \%$ Tween-20 (TBST). Antibodies for phosphorylated proteins were 
applied at $4^{\circ} \mathrm{C}$ overnight in 5\% BSA TBST, washed and visualized with a horseradish peroxidase conjugated anti-rabbit IgG secondary antibody (Cell Signalling \#7074S) and SuperSignal West Dura Chemiluminescent Substrate (Thermo Scientific \#34075). Images were acquired with a ChemiDoc Touch imager (BioRad). Membranes were stripped with Restore PLUS stripping buffer (Thermo Scientific \#46430). They were blocked and reprobed for antibodies to total proteins, and again striped and re-probed for GAPDH or $\beta$ actin to normalize for protein loading. All primary antibodies were monoclonal rabbit from Cell Signaling Technology. Catalog numbers and dilutions were as follows; Phosphorylated-Erk 1/2 (P-Erk) Y204(\#4377S, diluted 1:500), total Erk (\#4695, 1:500), Phospho-p38 (P-p38) T190/Y182 (\#4511, 1:500), total p38, (\#9212, total Axl (\#4939, 1:500), total Tyro3 (\#5585, 1:500), total MerTK (\#4319, 1:500), Sox2 (\# 3579, 1:500), Caspase-9 (\#9502, 1:1000), $\beta$-actin (\#4970, 1:2000), and GAPDH (\#2118, 1:2000). Images representative of biological replicates are shown and cropped for presentation. For P-Erk, P-p38, and p27 quantification, images from five independent experiments were quantified relative to each vehicle treated scrambled shRNA control with BioRad ImageLab software and then normalized to housekeeping gene expression. The P-Erk to P-p38 ratio was obtained by dividing the normalized P-Erk and P-p38 values for each independent experiment. All data are shown as fold change from control.

\section{Real time reverse transcriptase PCR (qRT-PCR)}

Cells were lysed and RNA was harvested using the Qiagen RNeasy kit followed by reverse transcription using Invitrogen SuperScript II Reverse Transcriptase. Real time qPCR was performed using TaqMan Universal PCR Master Mix and Gene Expression 
Assays on a Applied Biosystems ViiA 7 instrument. TaqMan MGB probes (Applied Biosystems) were as follows: MERTK (Hs00179024_m1), p27 / CDKN1B (Hs00153277), SOX2 (Hs01053049_s1) and NANOG (Hs02387400). We designed primers and a probe to specifically detect NR2F1 / TFCOUP1: forward; CAAAGCCATCGTGCTGTTCAC, reverse; CCTGCAGGCTCTCGATGT, and probe; TCAGACGCCTGTGGCCTG. $\beta$ actin (Hs01060665_g1) was used as an internal control for the normalization of target gene expression.

\section{Flow cytometry for histone post-translational modifications and Ki67.}

Cell pellets were fixed and permeabilized with dropwise addition of $1 \mathrm{ml}$ of cold $70 \%$ ethanol and then incubated overnight. All steps were at $4^{\circ} \mathrm{C}$ or on ice. Samples were then washed, blocked and incubated for one hour in flow buffer (PBS with 2\% FBS and $2 \mathrm{mM}$ EDTA) with each of the following antibodies; Alexa 647 conjugated rabbit anti histone H3 tri-methylated lysine 27 diluted 1:50 (Cell Signaling Technology \#12158), unconjugated rabbit polyclonal anti histone H3 tri-methylated lysine 9 (Abcam \#8898), or APC conjugated rabbit anti-human Ki-67 antibody (Biolegend \#350513). Cells were washed twice with flow buffer. The unconjugated histone H3 tri-methylated lysine 9 antibody was detected with an Alexa 647 conjugated anti-rabbit IgG diluted 1:250 (Cell Signaling Technology \#4414). Data was acquired with a three laser (405 nm, $488 \mathrm{~nm}$ and $640 \mathrm{~nm}$ ) Becton Dickinson FACS Aria IIu flow cytometer. Gating was forward scatter vs side scatter, single cells (linear on FSC-A vs FSC-H), then the 670/30 filter (APC or Alexa 647) vs forward scatter or histogram. An isotype control antibody was used for setting the gates. Negative and dim cells were selected for methylated histones. Negative 
cells were selected for Ki67. Data is presented as representative plots or mean \pm SEM of triplicate wells from replicate experiments.

\section{Cell cycle analysis}

Cells were cultured for three days as indicated and pulsed with $10 \mu \mathrm{M}$ bromodeoxyuridine (BrdU) for 30 minutes. The cells were collected with trypsin as necessary and then fixed and stained for total DNA with 7-AAD and BrdU incorporated into DNA using the Becton Dickinson APC BrdU flow kit (\#552598). Data was acquired with a Becton Dickinson FACS Aria IIu flow cytometer. Gating was forward scatter vs side scatter, single cells (linear on FSC-A vs FSC-H), then APC (BrdU) vs 7-AAD (DNA).

\section{Left ventricle intracardiac injection xenograft model of prostate cancer metastasis.}

Stable shRNA infected PC $3^{\mathrm{GFP}}\left(1 \times 10^{6}\right)$ or Du145 ${ }^{\mathrm{GFP}}\left(2 \times 10^{5}\right)$ prostate cancer cells were suspended in $100 \mu 1$ of PBS and injected into male CB.17. SCID mice (6-8 weeks of age: Charles River Labs) by left ventricle intracardiac injection. For analysis of metastasis free survival, bioluminescence images were acquired after injection of luciferin twice weekly using a PerkinElmer IVIS 2000 system. Animals that had a large portion of the signal in the lungs (indicative of a right ventricle injection) were removed from analysis a priori. After removing mice that had a right ventricular injections or did not survive the procedure, the following numbers of animals were analyzed; PC3 shControl; 6, PC3 shMER; 7, Du145 shControl; 20, and Du145 shMER; 18. Time to metastasis formation visible by bioluminescence (or death in rare cases) was then determined from the images. 
The data was analyzed by Kaplan-Meier analysis. For analysis of transit to the bone marrow, different mice, 5 mice per group, were sacrificed 24 hours after tumor cell injection, and their pelvis, femora and tibiae were harvested. The bones were crushed with a mortar and pestle and strained to remove debris. All steps used PBS buffer with $2 \%$ FBS unless otherwise noted. Cells were first depleted of mouse cells with a Mouse Cell Depletion Kit magnetic labeling system (Miltenyi Biotec \# 130-104-694) and antiBiotin MicroBeads and an AutoMACS machine (Miltenyi Biotec). The enriched cells were incubated with an APC-Cy7 conjugated anti-HLA-ABC antibody (BioLegend \#311426) and a PerCP-Cy5.5 conjugated anti-mouse lineage cocktail (CD3e, CD11b, B220, Ter-119, Ly-6G and Ly-6C) (BD Biosciences \#561317), for an hour at $4{ }^{\circ} \mathrm{C}$, washed and resuspended in PBS with $2 \%$ FBS, 2 mM EDTA and $0.5 \mu \mathrm{g} / \mathrm{ml}$ DAPI. Thereafter, the percentage of disseminated prostate cancer cells (DTCs) was determined by gating on single, viable, lineage negative, $\mathrm{HLA}^{+}$cells with a FACSAria IIu flow cytometer. Mice injected with PBS rather than PCa cells were used as a negative control for flow cytometry. Data represents 3 independent experiments.

\section{Prostate cancer subcutaneous tumor model.}

One million prostate cancer cells suspended in $50 \mu \mathrm{l}$ complete media were mixed with an equal volume of cold collagen solution and then slowly injected under the skin of the back of SCID mice; 5 mice per group. Bioluminescence images were acquired weekly. Animals were sacrificed before tumors grew to $1 \mathrm{~cm}^{3}$. All experimental procedures were approved by the University of Michigan Committee for the Use and Care of Animals. 


\section{Prostate Cancer Cell Viability / MTS Assay}

Prostate cancer cells were seeded at 2000 cells per well in 96 well plates and rested for one day in 10\% FCS RPMI media. The media was subsequently changed to the indicated serum concentrations and the cells were cultured for an additional 3 days. The total viable cell number was then assayed with the Cell Titer Aqueous One Solution MTS Proliferation Assay System (Promega \#G3580) by absorbance at $490 \mathrm{~nm}$. Data represent means of three independent experiments.

\section{Statistical analyses}

The type I error rate $(\alpha)$ was set to 0.05 for all analyses. Two-sample, two-tailed Student's t-tests were used to compare means of two groups. One-way repeated measures analysis of variance (ANOVA) with Bonferroni post-hoc testing was used for data normalized to housekeeping genes (blots and PCR). Standard one-way ANOVA with Tukey's Honest Significant Difference post-hoc testing was used for multiple comparisons in other experiments. The Log-rank test was used for Kaplan-Meier survival analyses. Growth curves for subcutaneous tumors were analyzed with a mixed design (split plot) ANOVA with repeated measures. All analyses were conducted with SPSS software, except for t-tests, which were performed in Microsoft Excel.

\section{RESULTS}

\section{MERTK knockdown causes dormancy associated changes in MAPK activity and} p27 expression. 
To study the importance of TAM signaling on dormancy, each TAM receptor was stably knocked down with shRNA in PC3, Du145 and C4-2B prostate cancer cell lines. Protein expression of each receptor was decreased by at least $80 \%$ (Figure S1). A decreased ratio of P-Erk 1/2 to P-p38 MAPK marks cellular dormancy in prostate and other cancers(Kobayashi, Okuda et al. 2011, Bragado, Estrada et al. 2013, Chery, Lam et al. 2014). Therefore, we first examined P-Erk1/2 and P-p38 levels in PC3 cells with each of the TAM receptors knocked down. The ratio of P-Erk 1/2 to P-p38 was significantly decreased in serum starved MERTK knockdown cells, but not in TYRO3 or AXL knockdown cells (Figure 1A and 1B). Similarly, the cell cycle inhibitor p27 was also previously found to be a dormancy marker(Kobayashi, Okuda et al. 2011, Bragado, Estrada et al. 2013). In agreement with the Erk and p38 data, we found higher basal p27 protein expression in the MERTK knockdown cells (Figure 1C and 1D). These data are consistent with a dormant phenotype in prostate cancer cells as a result of chronically reduced expression of MERTK.

\section{MERTK knockdown causes expression of dormancy and pluripotency associated transcription factors}

Transcription factors first studied in embryonic stem cells have been found to promote cancer dormancy(Sosa, Parikh et al. 2015). Therefore, we determined the basal expression level of three of these transcription factors in PC3 cells with each of the TAM receptors knocked down by shRNA. In parallel with the MAPK and p27 data, we saw marked upregulation of SOX2 message and protein in shMER but not shAXL or 
shTYRO3 cells (Figure 2A and 2B). Similarly, we also observed increased expression of SOX2 in shMER C4-2B cells (Figure 2A). We also observed increased NR2F1 and NANOG mRNA in shMER but not shAXL or shTYRO3 cells (Figure 2C and 2D). Because of the possibility of off target effects of shRNAs, we performed analogous studies with siRNA rather than shRNA and found that a siRNA targeting MERTK increased expression of SOX2 and NANOG in PC3 and C4-2B cells.

\section{MERTK knockdown induces cell cycle changes associated with cellular dormancy.}

Cellular dormancy and decreased Erk $1 / 2$ activity are also characterized by arrest in the G1 and G0 phases of the cell cycle(Aguirre-Ghiso, Ossowski et al. 2004). Therefore, we compared cell cycle characteristics of TAM receptor knockdown PC3 cells cultured in $0.1 \%$ serum using flow cytometry to detect antibody labeled pulsed bromodeoxyuridine (BrdU) incorporated into DNA and 7-AAD to quantify total DNA content. This assay identifies BrdU positive cells as S-phase, less than 2n DNA as apoptotic or necrotic cells, 2n BrdU negative cells as G1 and G0 phases, 4n BrdU negative cells as G2 and M phases and cells with $>4$ DNA as polyploid (Figure 3A). In agreement with our other data, MERTK knockdown cells showed a pattern consistent with dormancy, with a higher percentage of cells in G0/G1 and lower percentage in G2/M compared to control (Figure 3B). Curiously, TYRO3 knockdown cells showed the opposite pattern with fewer cells in G0/G1 and more cells in the G2 and M phases. We did not convincingly see other analogous results for TYRO3 in our other experiments. We did not see differences between the different TAM knockdown cells in the percentage of cells in S-phase but 
note that there are very few cells in $S$ phase in this study because of the low serum conditions. However, we did observe a reduction in the sub-G0 (apoptotic and necrotic) population in the shMER cells. Further, western blots showed decreased levels of cleaved Caspase-9 in the shMER cells, thus suggesting that this reduced sub-G0 population represented reduced apoptosis. There is precedence in the literature for correlation of reduced apoptosis with a dormant phenotype(Aguirre-Ghiso, Ossowski et al. 2004). Further, p38 stimulated cellular dormancy and reduced apoptosis have been proposed to be adaptive responses to allow DTCs to survive when conditions are not conducive to growth(Ranganathan, Adam et al. 2006).

\section{Depletion of MERTK increases metastasis free survival in vivo}

We next tested the importance for MERTK for dormancy escape in vivo. GFP and luciferase labeled control shRNA or MERTK shRNA PC3 or Du145 PCa cells were injected in the left ventricle of SCID mice and time to metastases visible by bioluminescence imaging, or death in rare cases, was evaluated with Kaplan-Meier analysis (Figure 4A). Metastases are primarily to bone with both cell lines. Metastasis free survival in this model is established in the literature as a measure of dormancy (Kobayashi, Okuda et al. 2011). In agreement with the in vitro data, metastasis free survival was prolonged in mice injected with shMER PC3 cells relative to control cells (Figure 4B, top left). With Du145 cells, the increase in metastasis free survival with MERTK knockdown was small and not statistically significant (Figure 4B, lower left). However, we noted that shMER Du145 cells appeared to develop metastases to the head 
more slowly, which approached statistical significance (Figure 4B, lower right). Time to cranial metastases was again significantly different with PC3 cells. A defect in transit to the bone marrow could also explain delayed metastasis formation but would not involve dormancy escape. Therefore, in separate experiments, we used flow cytometry to quantify the percentage of control or shMER PC3 cells in marrow one day after injection and found no difference (Figure 4C-D). This further supports the conclusion that MERTK is selectively important for dormancy escape rather than transit to the bone marrow in this model.

\section{MERTK knockdown does not decrease prostate cancer growth in vitro or at a} subcutaneous site in vivo.

We hypothesized that the role of MERTK was somewhat specific to cellular dormancy and therefore it should not greatly affect overall cellular growth in vitro or in vivo at a site not dependent on the usual microenvironment, such as a subcutaneous site with an artificial extracellular matrix. Indeed, other groups who have derived dormant and tumorigenic cancer cell lines have observed similar growth in culture(Bragado, Estrada et al. 2013, Sharma, Xing et al. 2016). In agreement with these expectations, we saw no difference in subcutaneous growth in vivo between control and shMER PC3 cells and saw slightly higher growth of Du145 shMER cells compared to control (figure 5A). Similarly, we saw no significant differences in relative cell number, as measured by MTS assay, between any of the TAM shRNA knockdowns in PC3, Du145 and C4-2B cells cultured for 3 days in $0.1 \%, 1 \%$ or $10 \%$ serum (Figure 5B). The Du145 shMER cells trended 
towards higher relative cell number but did not reach statistical significance after multiple comparison testing.

\section{Cell cycle changes of MERTK knockdown are MAP kinase dependent.}

Because of the well-established role of MAP kinases in regulation of cancer cellular dormancy, we investigated if p38 was required for the dormancy associated cell cycle changes induced by knockdown of MERTK. In appreciation of the known role of epigenetic changes in dormancy regulation and the time period required for these changes to occur, we cultured control and MERTK shRNA PC3 and Du145 cells with p38 inhibitor SB203580 or $0.05 \%$ DMSO solvent control for two weeks before performing experiments(Sosa, Parikh et al. 2015). The expected compensatory increase in P-p38 in response to p38 active site inhibition was observed by Western blot (Figure S2A). We again observed an increased percentage of G0/G1 and decreased percentage of G2/M cells with MERTK knockdown both in PC3 and Du145 cells (Figure 6). This change induced by MERTK knockdown was completely reversed by p38 inhibition, thus showing involvement of MAP kinases. Similarly, we also observed a increased percentage of Ki67 negative cells (non-cycling) with MERTK knockdown in PC3 cells, which was also reversed by p38 inhibition (Figure S2C). Curiously, we did not observe reversal of shMER induced p27 upregulation with p38 inhibition (Figure S2B). This suggests that not all of the effects of MERTK knockdown are MAP kinase dependent. 


\section{MERTK knockdown induces dormancy associated changes in histone $\mathbf{H 3}$ methylation by a MAP kinase dependent mechanism.}

Lastly, we used flow cytometry with specific antibodies to determine the effect of shRNA knockdown in PC3 cells of each of the TAM receptors on histone H3 tri-methylated lysine 9 (H3 K9 me3) and histone H3 tri-methylated lysine 27 (H3 K27 me3). Both of these histone marks are increased in dormant cells(Sosa, Parikh et al. 2015). Because the majority of cells were positive, we gated on the negative and dim populations rather than the positive population (Figure 7A). We observed the expected dormancy associated change in $\mathrm{H} 3 \mathrm{~K} 9 \mathrm{me} 3$, and a trend towards significance for $\mathrm{H} 3 \mathrm{~K} 27 \mathrm{me} 3$ with MERTK knockdown but no significant differences for AXL or TYRO3 knockdown (Figure 7B). In cells treated with the p38 inhibitor SB203580 or solvent control, we saw significant dormancy associated changes with MERTK knockdown, which were partially reversed by p38 inhibition (Figure 7C). DISCUSSION

Overall, these studies implicate MERTK in prostate cancer dormancy escape through a MAP kinase dependent mechanism linked to transcriptional and epigenetic regulation. Knockdown of MERTK consistently induced the changes expected for dormant cells; a decreased ratio of P-Erk to P-p38, increased p27 expression, expression of dormancy and pluripotency associated transcription factors, and G0/G1 arrest. Further, these findings translated to an increased metastasis free survival in vivo. This identifies MERTK as being important for the process whereby one or a few cancer cells progress to a small tumor (i.e. escape from cellular dormancy). As expected, MERTK knockdown did not 
inhibit growth in culture or growth of subcutaneous tumors implanted in an artificial matrix. This lack of a general growth inhibitory effect of MERTK knockdown suggests specificity for dormancy regulation in bone and a requirement for a signal from the microenvironment rather than dysregulation of multiple cellular processes.

Our data do not identify which signal(s) from the microenvironment interact with MERTK to regulate dormancy. The four other TAM receptor ligands, other than GAS6 (Tulp1, Tubby, Galectin 3 and Protein S), may also play a role. Although not a MERTK ligand, retinoic acid may be indirectly involved as well because it has been shown to stimulate cancer dormancy through NR2F1 and also interacts indirectly with MERTK in immune cells(Garabuczi, Sarang et al. 2015, Sosa, Parikh et al. 2015). Because no recombinant protein or other treatment other than low serum conditions was required for our observed in vitro effects, knockdown of MERTK may have caused epigenetic reprogramming of the cells as a result of chronic alterations in signaling from autocrine TAM receptor ligands. Prostate cancer cells are known to express MERTK ligands GAS6 and PROS1 (Protein S)(Jung, Decker et al. 2016, Ning, Zhong et al. 2016). The observed changes in histone $\mathrm{H} 3$ methylation also suggests that part of the effects of MERTK may be due to epigenetic reprogramming rather than through a more immediate response to ligands.

Previously, we examined the expression of TYRO3 and AXL, but not MERTK in disseminated tumor cells (DTCs) vs prostate cancer primary tumors and gross metastases(Taichman, Patel et al. 2013). We reported higher expression of AXL in DTCs 
and higher expression of TYRO3 in primary tumors and gross metastases. Thus, we hypothesized that AXL might be important for maintenance of dormancy and TYRO3 might be important for escape from dormancy or growth of a gross tumor. In retrospect, it would have been useful in include MERTK in these studies. Our most recent work, currently in press, confirms the earlier hypothesis regarding AXL and shows that AXL is required for TGF- $\beta 2$ induced prostate cancer dormancy(Yumoto 2016). However, prior to the current work, the contribution of MERTK to prostate cancer dormancy remained completely unclear. Here, we took an unbiased approach and were consistently guided by the data to focus on MERTK. However, our data does not rule out contributions from AXL or TYRO3 in stimulation of dormancy escape. For example, the percent knockdown might have been insufficient to observe effects with shAXL or shTYRO3.

We recently reported a role for MERTK in formation of prostate cancer stem-like cells (CSCs)(Jung, Decker et al. 2016, Shiozawa, Berry et al. 2016). However, the proliferative rate of CSCs was not examined in these studies and other literature reports that CSCs can be either slowly or rapidly cycling(Sharma, Xing et al. 2016, Takeishi and Nakayama 2016). Additionally, others identified MERTK in an unbiased screen of over 100 kinases as a stimulator of prostate cancer metastasis. Therefore, our data showing a new role for MERTK in dormancy escape combined with the other studies implicating MERTK in CSC formation and metastasis, suggests that MERTK plays a role in prostate cancer progression by regulating several processes. 
In summary, we are beginning to understand the roles of the TAM kinases in prostate cancer dormancy regulation. AXL plays a role in dormancy maintenance through TGF- $\beta 2$ and microenvironment dependent effects. The role of TYRO3 remains less clear. Conversely this study provides the first evidence that MERTK stimulates prostate cancer dormancy escape. Our studies with a p38 inhibitor show MAP kinases are required for MERTK to stimulate dormancy escape. Furthermore, MERTK causes changes observed in histone $\mathrm{H} 3$ methylation, pluripotency associated transcription factors and cell cycle regulatory proteins observed in dormancy regulation of other cancers (Figure 8). Thus, MERTK appears to reprogram disseminated tumor cells to grow rather than remain dormant. This understanding of the role of MERTK should inform future studies of prostate cancer dormancy and may provide a therapeutic target for prevention of recurrence.

\section{ACKNOWLEDGEMENTS}

The authors wish to thank Taocong Jin with assistance with primer design and qRT-PCR. Portions of figure 8 are derived from the Servier powerpoint image bank.

\section{References}

Aguirre-Ghiso, J. A., L. Ossowski and S. K. Rosenbaum (2004). "Green fluorescent protein tagging of extracellular signal-regulated kinase and p38 pathways reveals novel dynamics of pathway activation during primary and metastatic growth." Cancer Res 64(20): 7336-7345.

Amling, C. L., M. L. Blute, E. J. Bergstralh, T. M. Seay, J. Slezak and H. Zincke (2000). "Long-term hazard of progression after radical prostatectomy for clinically localized prostate cancer: continued risk of biochemical failure after 5 years." J Urol 164(1): 101105. 
Bragado, P., Y. Estrada, F. Parikh, S. Krause, C. Capobianco, H. G. Farina, D. M. Schewe and J. A. Aguirre-Ghiso (2013). "TGF-beta2 dictates disseminated tumour cell fate in target organs through TGF-beta-RIII and p38alpha/beta signalling." Nat Cell Biol 15(11): 1351-1361.

Caberoy, N. B., Y. Zhou and W. Li (2010). "Tubby and tubby-like protein 1 are new MerTK ligands for phagocytosis." EMBO J 29(23): 3898-3910.

Chery, L., H. M. Lam, I. Coleman, B. Lakely, R. Coleman, S. Larson, J. A. AguirreGhiso, J. Xia, R. Gulati, P. S. Nelson, B. Montgomery, P. Lange, L. A. Snyder, R. L. Vessella and C. Morrissey (2014). "Characterization of single disseminated prostate cancer cells reveals tumor cell heterogeneity and identifies dormancy associated pathways." Oncotarget 5(20): 9939-9951.

Faltermeier, C. M., J. M. Drake, P. M. Clark, B. A. Smith, Y. Zong, C. Volpe, C. Mathis, C. Morrissey, B. Castor, J. Huang and O. N. Witte (2015). "Functional screen identifies kinases driving prostate cancer visceral and bone metastasis." Proc Natl Acad Sci U S A.

Garabuczi, E., Z. Sarang and Z. Szondy (2015). "Glucocorticoids enhance prolonged clearance of apoptotic cells by upregulating liver $X$ receptor, peroxisome proliferatoractivated receptor-delta and UCP2." Biochim Biophys Acta 1853(3): 573-582.

Graham, D. K., D. DeRyckere, K. D. Davies and H. S. Earp (2014). "The TAM family: phosphatidylserine sensing receptor tyrosine kinases gone awry in cancer." Nat Rev Cancer 14(12): 769-785.

Jung, Y., A. M. Decker, J. Wang, E. Lee, L. A. Kana, K. Yumoto, F. C. Cackowski, J. Rhee, P. Carmeliet, L. Buttitta, T. M. Morgan and R. S. Taichman (2016). "Endogenous GAS6 and Mer receptor signaling regulate prostate cancer stem cells in bone marrow." Oncotarget.

Jung, Y., Y. Shiozawa, J. Wang, N. McGregor, J. Dai, S. I. Park, J. E. Berry, A. M. Havens, J. Joseph, J. K. Kim, L. Patel, P. Carmeliet, S. Daignault, E. T. Keller, L. K. McCauley, K. J. Pienta and R. S. Taichman (2012). "Prevalence of prostate cancer metastases after intravenous inoculation provides clues into the molecular basis of dormancy in the bone marrow microenvironment." Neoplasia 14(5): 429-439.

Kobayashi, A., H. Okuda, F. Xing, P. R. Pandey, M. Watabe, S. Hirota, S. K. Pai, W. Liu, K. Fukuda, C. Chambers, A. Wilber and K. Watabe (2011). "Bone morphogenetic protein 7 in dormancy and metastasis of prostate cancer stem-like cells in bone." J Exp Med 208(13): 2641-2655.

Lee, E., A. M. Decker, F. C. Cackowski, L. A. Kana, K. Yumoto, Y. Jung, J. Wang, L. Buttitta, T. M. Morgan and R. S. Taichman (2016). "Growth Arrest-Specific 6 (GAS6) Promotes Prostate Cancer Survival by G1 Arrest/S Phase Delay and Inhibition of Apoptotic Pathway During Chemotherapy in Bone Marrow." J Cell Biochem. 
Morgan, T. M., P. H. Lange, M. P. Porter, D. W. Lin, W. J. Ellis, I. S. Gallaher and R. L. Vessella (2009). "Disseminated tumor cells in prostate cancer patients after radical prostatectomy and without evidence of disease predicts biochemical recurrence." Clin Cancer Res 15(2): 677-683.

Ning, P., J. G. Zhong, F. Jiang, Y. Zhang, J. Zhao, F. Tian and W. Li (2016). "Role of protein $\mathrm{S}$ in castration-resistant prostate cancer-like cells." Endocr Relat Cancer 23(8): 595-607.

Ranganathan, A. C., A. P. Adam, L. Zhang and J. A. Aguirre-Ghiso (2006). "Tumor cell dormancy induced by p38SAPK and ER-stress signaling: an adaptive advantage for metastatic cells?" Cancer Biol Ther 5(7): 729-735.

Sharma, S., F. Xing, Y. Liu, K. Wu, N. Said, R. Pochampally, Y. Shiozawa, H. K. Lin, K. C. Balaji and K. Watabe (2016). "Secreted Protein Acidic and Rich in Cysteine (SPARC) Mediates Metastatic Dormancy of Prostate Cancer in the Bone." J Biol Chem.

Shiozawa, Y., J. E. Berry, M. R. Eber, Y. Jung, K. Yumoto, F. C. Cackowski, H. J. Yoon, P. Parsana, R. Mehra, J. Wang, S. McGee, E. Lee, S. Nagrath, K. J. Pienta and R. S. Taichman (2016). "The marrow niche controls the cancer stem cell phenotype of disseminated prostate cancer." Oncotarget.

Shiozawa, Y., E. A. Pedersen, L. R. Patel, A. M. Ziegler, A. M. Havens, Y. Jung, J. Wang, S. Zalucha, R. D. Loberg, K. J. Pienta and R. S. Taichman (2010). "GAS6/AXL axis regulates prostate cancer invasion, proliferation, and survival in the bone marrow niche." Neoplasia 12(2): 116-127.

Sosa, M. S., F. Parikh, A. G. Maia, Y. Estrada, A. Bosch, P. Bragado, E. Ekpin, A. George, Y. Zheng, H. M. Lam, C. Morrissey, C. Y. Chung, E. F. Farias, E. Bernstein and J. A. Aguirre-Ghiso (2015). "NR2F1 controls tumour cell dormancy via SOX9- and RARbeta-driven quiescence programmes." Nat Commun 6: 6170.

Taichman, R. S., L. R. Patel, R. Bedenis, J. Wang, S. Weidner, T. Schumann, K. Yumoto, J. E. Berry, Y. Shiozawa and K. J. Pienta (2013). "GAS6 receptor status is associated with dormancy and bone metastatic tumor formation." PLoS One 8(4): e61873.

Takeishi, S. and K. I. Nakayama (2016). "To wake up cancer stem cells, or to let them sleep, that is the question." Cancer Sci 107(7): 875-881.

Yumoto, K., Matthew Eber, Jincheng Wang, Frank Cackowski, Eunsohl Lee, Ana Rita Nobre, Julio Aguirre-Ghiso, Younghun Jung, and Russell Taichman (2016). "Axl is required for TGF- $\beta 2$-induced dormancy of prostate cancer cells in the bone marrow." Scientific Reports (In press). 


\section{FIGURE LEGENDS}

Figure 1. TAM receptor knockdown and dormancy associated pathways in prostate cancer cells. A, Representative Western blots of PC3 cells with each of the TAM receptors knocked down by shRNA probed for phosphorylated or total p38 and Erk 1/2 or housekeeping genes. B, Quantification of the samples in panel A for P-Erk, P-p38 or the ratio of P-Erk to P-p38 relative to the values for the scrambled shRNA control and normalized to the housekeeping genes. $\mathbf{C}$, Representative Western blot for p27 of the same cells. D, Quantification of the data in C. All data is presented normalized to control. Error bars represent mean \pm SEM. * Represents $p<0.05$ compared to shRNA control cells.

Figure 2. TAM receptor knockdown and expression of dormancy and pluripotency associated transcription factors. A, PC3 cells with each of the TAM receptors knocked down by shRNA or C4-2B cells with MERTK knocked down, analyzed for SOX2 expression by qPCR. B, Representative SOX2 Western blot of shControl and shMER PC3 cells. C, D, PC3 TAM receptor shRNA cells with expression of NR2F1 or NANOG respectively, quantified by qPCR. E, PC3 cells with MerTK knocked down by siRNA and quantified for expression of SOX2 and NANOG by qPCR. F, C4-2B cells with MERTK knocked down by siRNA and quantified for expression of SOX2 and NANOG by qPCR. All data is presented normalized to control. Error bars represent mean $\pm \mathrm{SEM}$.

* Represents $p<0.05$ compared to shRNA or siRNA control cells. 
Figure 3. Cell cycle analysis of TAM receptor knockdown PC3 cells by flow cytometry with BrdU and total DNA labeling. A, Example flow plots for each cell type. Top: histograms of total DNA content labeled with7-AAD. Bottom: BrdU vs. total DNA plots. The significance of each population is as follows: < 2n DNA and BrdU negative; Sub-G0 (apoptotic and necrotic), 2n DNA and BrdU negative; $\mathrm{G}_{0}$ and G1 phases, $4 \mathrm{n}$ DNA and BrdU negative; $\mathrm{G}_{2}$ and $\mathrm{M}$ phases. BrdU positive; $\mathrm{S}$ phase, $>4 \mathrm{n}$ DNA; polyploid cells. $\mathrm{B}$, Quantification of the above cell cycle data. The table lists $p$-values for each cell type compared to control with significant comparisons marked with an asterisk. Error bars are shown for the G0G1 and G2M populations and represent mean \pm SEM. * Represents $p<$ 0.05 compared to shRNA control cells. $\mathbf{C}$, Western blots for total caspase-9 to verify the changes in Sub-G0 cells observed by flow cytometry.

Figure 4. MERTK knockdown and metastasis free survival in a prostate cancer left ventricle injection xenograft model. A, Experimental design. B, Kaplan-Meier analysis of time to formation of metastases visible by bioluminescence imaging or death in mice injected with luciferase labeled control or shMER PC3 or Du145 cells. Left panels: metastases to any site. Right panels: cranial metastases only.* Indicates $p<0.05$ vs control cells. C, Strategy for quantification of the percentage of DTCs in mouse bone marrow by flow cytometry after first depleting the number of mouse cells with immunomagnetic beads. D, Comparison of the percentage of DTCs in mouse bone marrow in control vs. shMER PC3 cells one day after intracardiac injection. Error bars represent mean \pm standard error. 
Figure 5. Effect of MERTK knockdown on growth of prostate cancer cells in culture and in subcutaneous tumors. A, Subcutaneous tumor growth of control or shMER PC3 or Du145 cells as measured by bioluminescence imaging. B, Relative cell number quantified by XTT assay after 3 days of culture in $10 \%, 1 \%$ or $0.1 \%$ serum for PC3, Du145 and C4-2B cells with each of the TAM receptors knocked down. Data are presented as mean \pm SEM. No pairwise comparisons were statistically significant.

Figure 6. Reversal of the shMER cell cycle phenotype with long term p38 inhibition. A, Control or shMER PC3 cells cultured with $5 \mu \mathrm{M}$ SB203580 or $0.05 \%$ DMSO solvent control for 14 days in $10 \%$ serum and 3 days in $0.1 \%$ serum followed by cell cycle analysis with total DNA and BrdU labeling as described for figure 3. Top: Total DNA flow cytometry histograms. Bottom: quantified data. Error bars are shown for the G0G1 and G2M populations and represent mean \pm SEM. * Represents $p<0.05$ for comparisons of interest. B, Cell cycle analysis as above of Du145 cells grown for 17 days in $10 \%$ serum with or without SB203580.

Figure 7. Presence and MAP kinase dependence of dormancy associated histone H3 post translational modifications in TAM receptor knockdown prostate cancer cells. A, Example plots for percentage of cells negative or dim for histone H3 tri-methylated lysine 9 and tri-methylated lysine 27 evaluated by flow cytometry. B, Percent of PC3 cells with each TAM receptor knocked down by shRNA negative for each histone H3 trimethylation. C, Control or shMER PC3 cells cultured for 14 days with or without p38 inhibitor SB203580 (as in figure 6) negative or dim for tri-methylated histone H3 lysine 9 
or lysine 27. Error bars represent mean \pm SEM. * Represents $p<0.05$ compared to control for panel B, or for comparisons of interest for panel C.

Figure 8. Results summary 
Figure 1

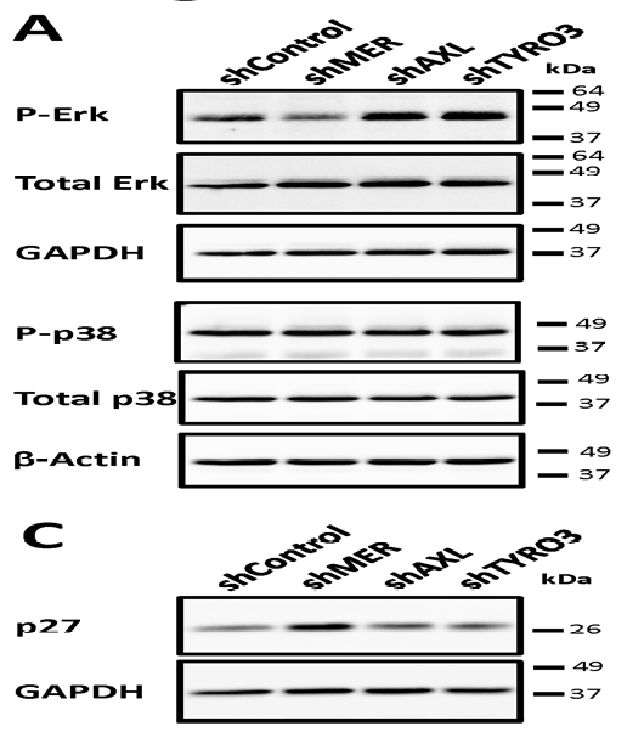

B
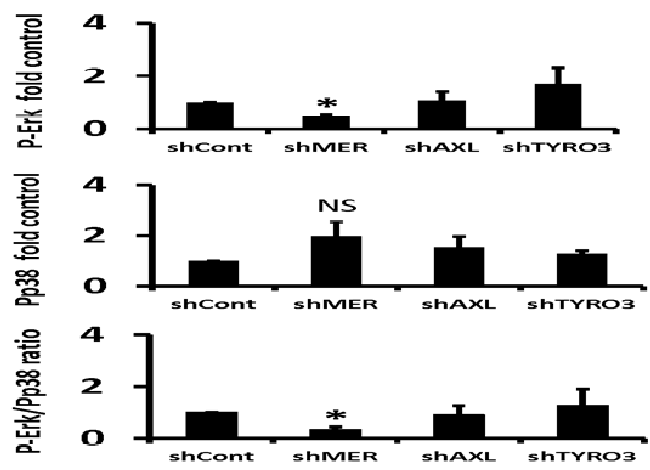

D

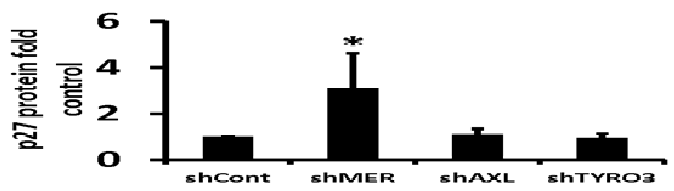

fig 1 . 
Figure 2

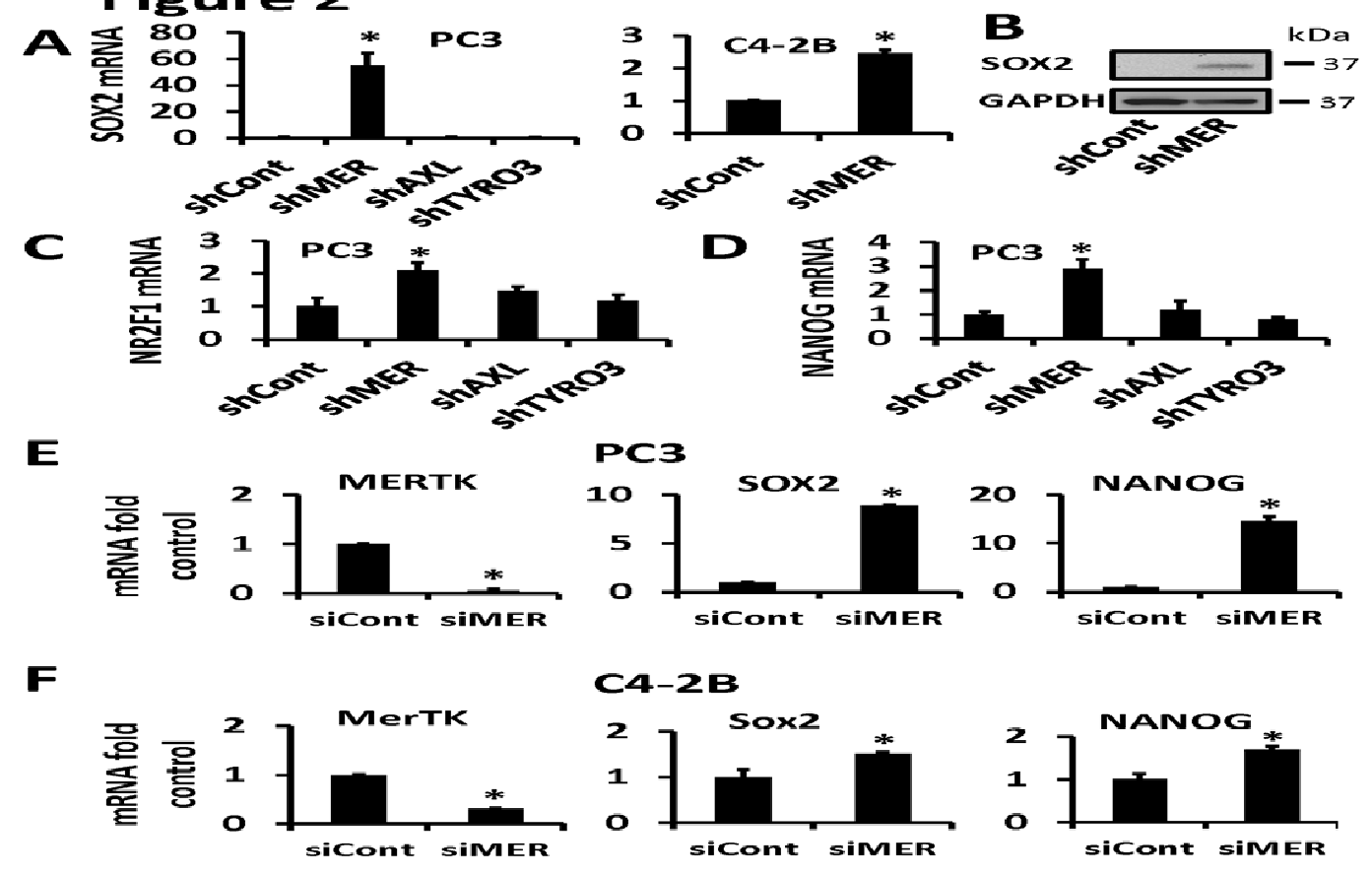

fig 2 . 


\section{Figure 3}

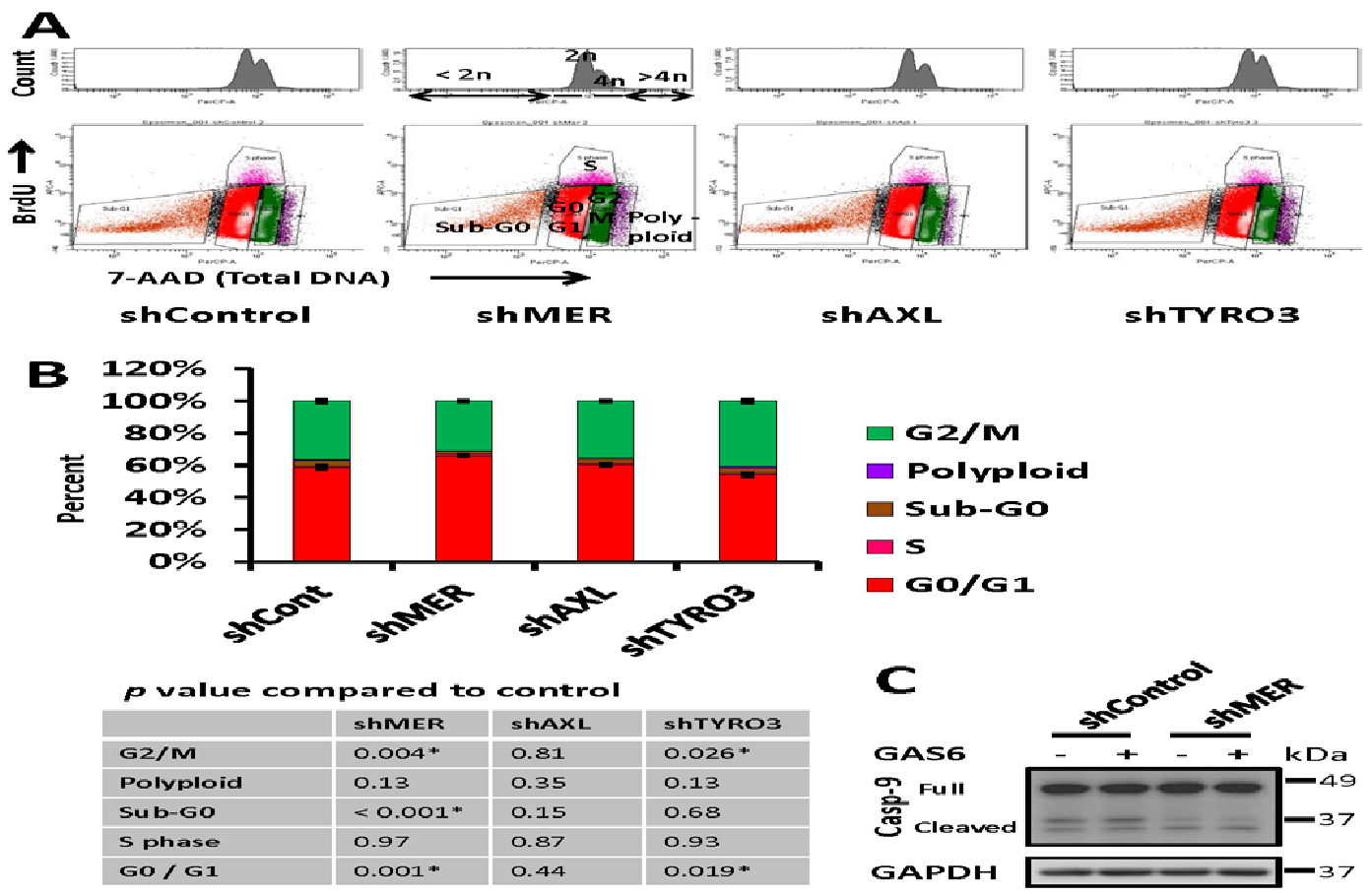

fig 3 . 
Figure 4

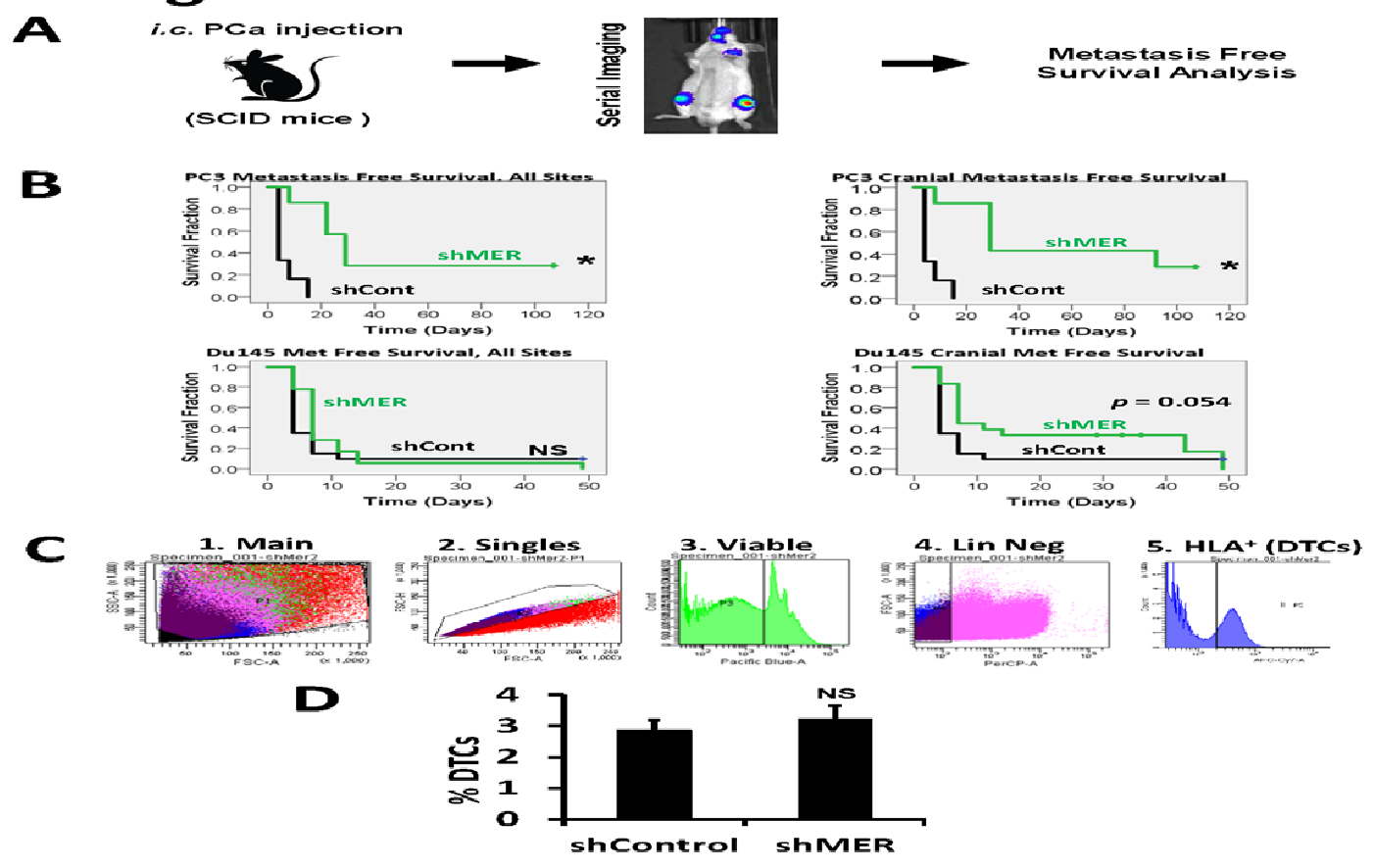

fig 4 . 


\section{Figure 5}

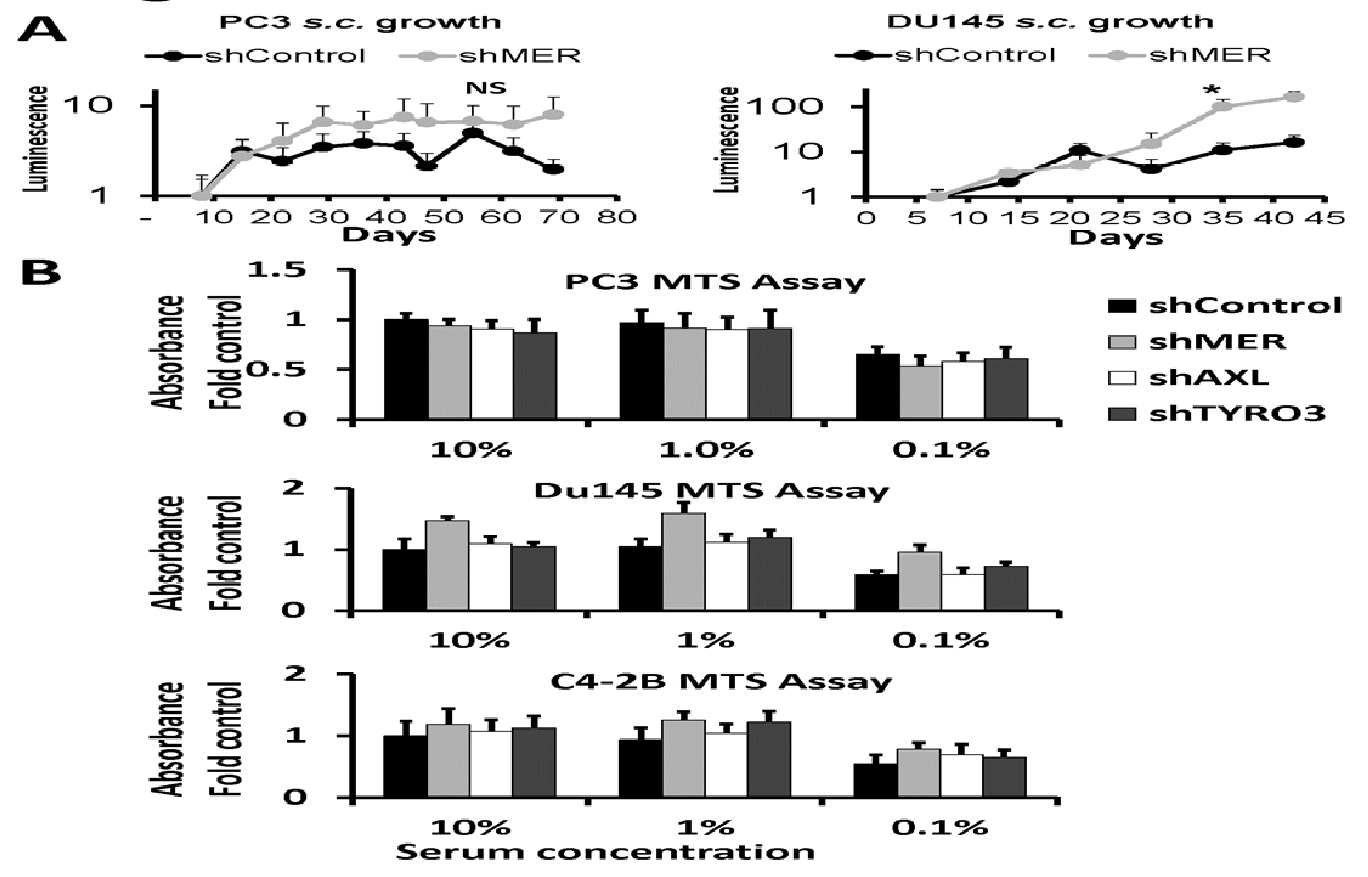

fig 5 . 


\section{Figure 6}
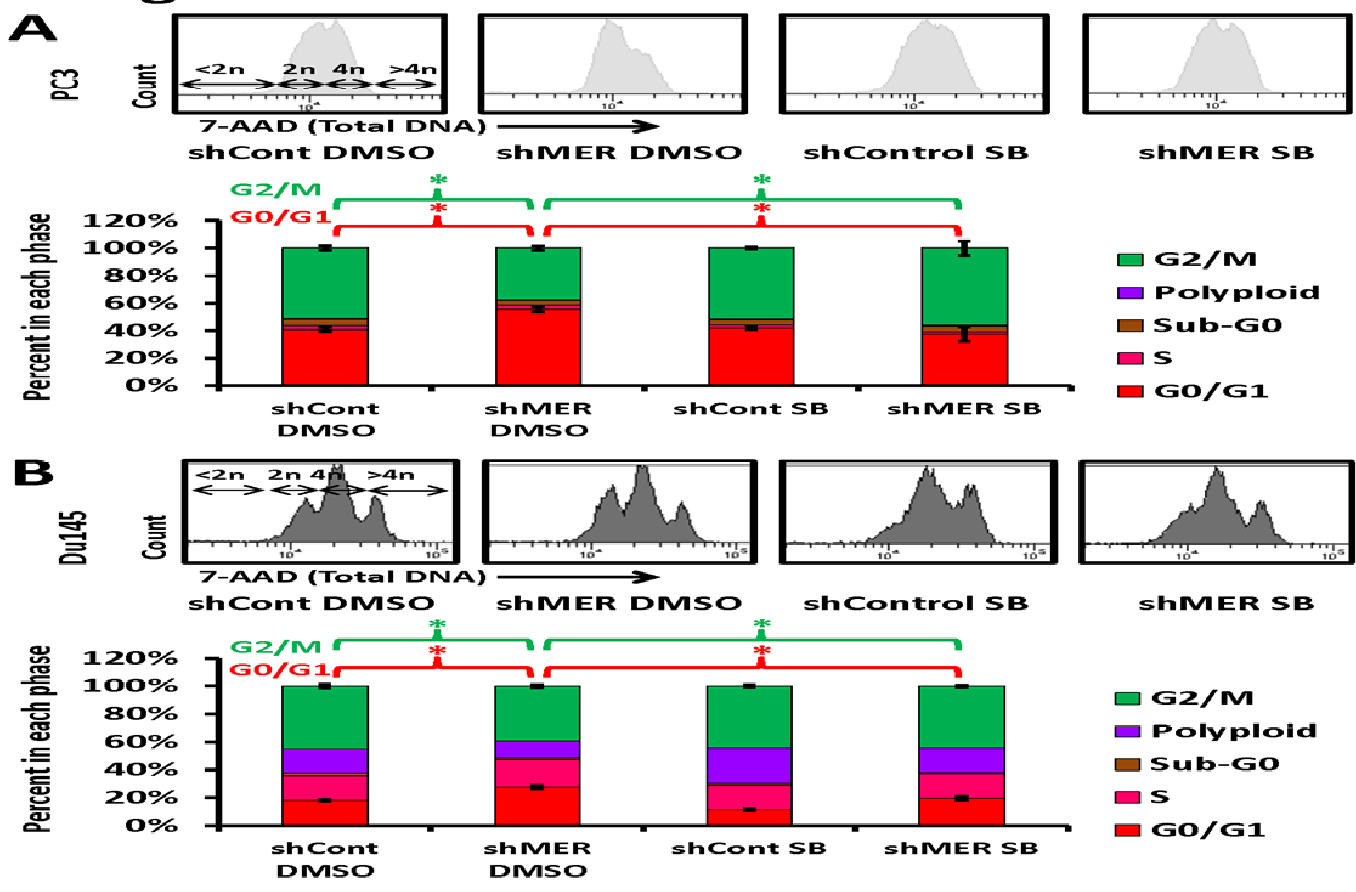

fig 6 . 
Figure 7
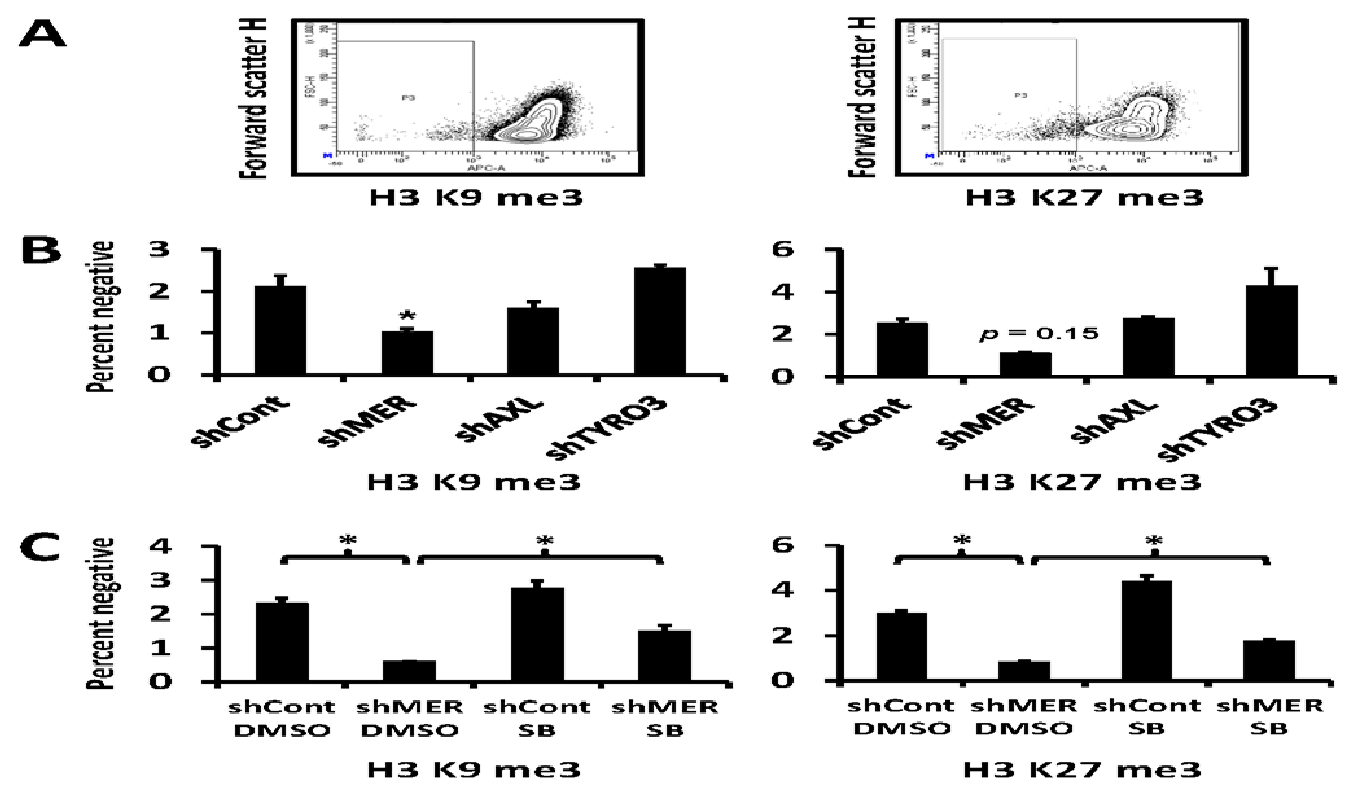

fig 7 . 


\section{Figure 8}

\section{MERTK}

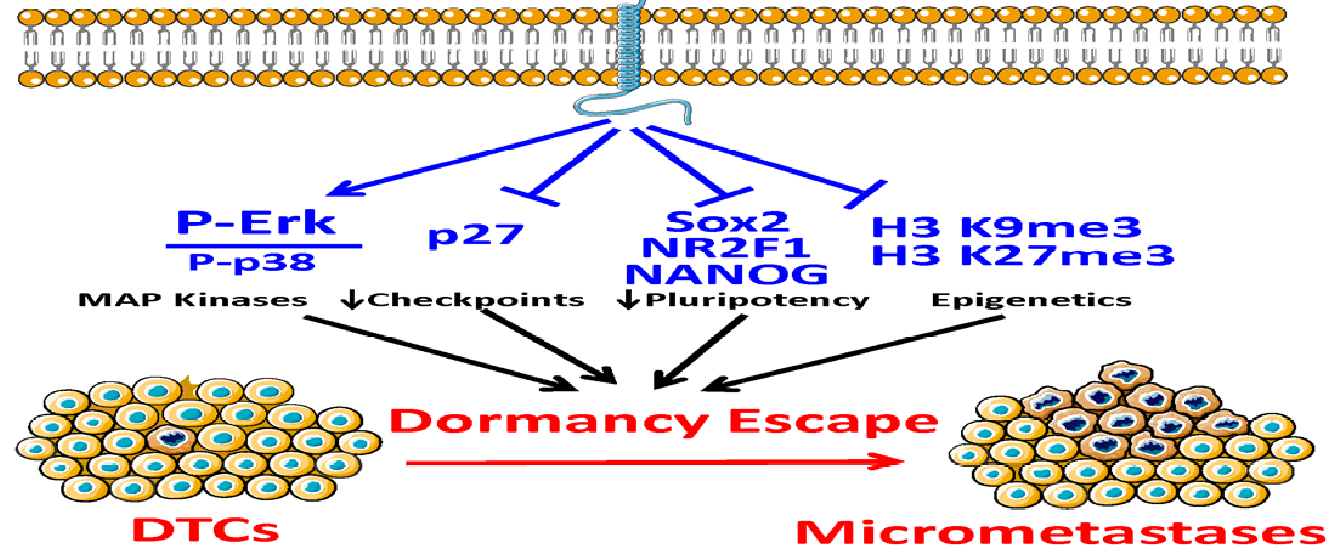

fig 8 . 
Figure S1
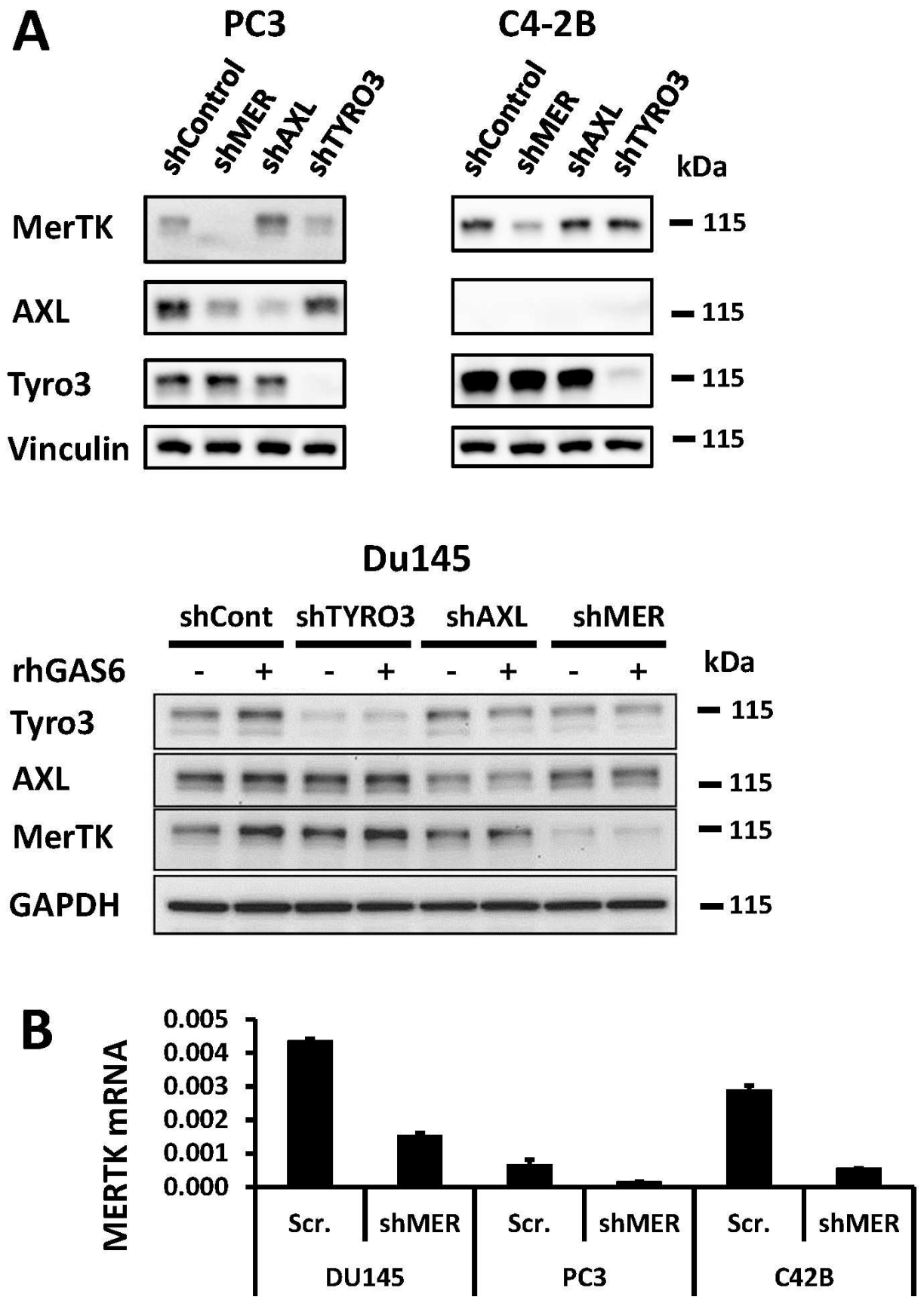

fig S1 . 
fig S2 . 
graphical abstract image . 